\title{
Self management of long term oral anticoagulation was as effective as specialist anticoagulation clinic management
}

\author{
Cromheecke ME, Levi M, Colly LP, et al. Oral anticoagulation self-management and management by a specialist \\ anticoagulation clinic: a randomised cross-over comparison. Lancet 2000 Jul 8;356:97-102.

\section{QUESTION: In patients needing long term oral anticoagulation treatment, is self management as effective as specialist anticoagulation clinic management?}

Design

6 month, randomised \{allocation concealed*\}†, controlled crossover trial with blinded outcome assessments (patients and clinicians)*.

\section{Setting \\ Anticoagulation clinic in the Netherlands.}

\section{Patients}

50 self supporting patients (mean age 42 y, 59\% men) who were receiving long term anticoagulation treatment with oral agents. 49 patients (98\%) completed the study.

\section{Intervention}

Self managed care involved education about the principles and monitoring of treatment. Live and video instructions were given on how to use a capillary fingerstick and automated device for self measurement. Patients were then instructed about dose adjustments of oral anticoagulants by using a standard nomogram. Anticoagulation clinic management included measurements and dosing done at the clinic. After 3 months, patients crossed over to the other management group for 3 more months.

\section{Main outcome measures}

The primary outcome was number of measurements within 0.5 international normalised ratio (INR) units of the therapeutic target. Secondary outcomes were percentage of time in the target range, number of patients in the target range, and number of patients who achieved better control of anticoagulation during either period.

\section{Main results}

The number of self managed measurements within 0.5 units of the target INR did not differ from clinic management $(55 \% v 49 \%,\{95 \%$ CI for the $6 \%$ difference $0.06 \%$ to $12 \%\} \ddagger, \mathrm{p}=0.06)$. 29 patients during self management $(60 \%)$ spent $>50 \%$ of the time in the target range compared with 25 patients $(52 \%)$ during clinic management $(\mathrm{p}<0.05)$. Of the 49 patients completing the study, $34(70 \%)$ had better control of anticoagulation during self management, $10(20 \%)$ had better control during clinic management, and $5(10 \%)$ showed no difference.

\section{Conclusion}

In patients needing long term oral anticoagulation treatment, self management was as effective as specialist anticoagulation clinic management.
*See glossary.

$\dagger$ Information provided by author.

$\ddagger$ CI calculated from data in article.

\section{COMMENTARY}

Patients who need long term anticoagulation with vitamin K antagonists must submit to regular prothrombin time (INR) testing, a routine that is at least a nuisance and often at odds with home and work schedules. More patient centred approaches are needed. Patients readily learn how to self test and how to self adjust the warfarin dosage. The therapeutic accuracy of self management was similar to that of physicians in general practice. ${ }^{1}$ In this randomised crossover study by Cromheecke et al, Dutch patients seem to be as good as the specialists and the computer algorithms that constitute "standard care." Consistent but indirect evidence suggests that anticoagulation clinics are the gold standard of anticoagulant care, ${ }^{2}$ and it appears that patients can become experts in managing their own warfarin treatment.

The results of this relatively small study rest on sound methods. Even if the accuracy of either approach to dosing was overstated or understated, the clinical message would be the same. On their own, selected patients do quite well. But how were they selected? Consecutive patients were enrolled, mitigating the possibility of selection bias. These patients, who were described as "self supporting," may be unusually well educated or self reliant. Self managed patients and anticoagulation clinics were tested often, every 1 to 2 weeks. In addition to patient ability and testing frequency, the costs of home testing limit the generalisability of this study. Insurance companies in the US, including Medicare, are as yet unwilling to subsidise this technology.

If self management is sufficiently accurate, does it also limit bleeding and thromboembolic events? At present, we do not know the answer, and only large studies could find a real difference in rates of relatively rare complications. Nevertheless, the convenience factor is important. Patients and physicians can opt for home testing and patient self dosing. Unfortunately, the choice is difficult because of cost, not because of clinical validity or applicability.

Daniel Becker, MD, MPH University of Virginia Medical Center Charlottesville, Virginia, USA

1 Sawicki PT. A structured teaching and self-management program for patients receiving oral anticoagulation: a randomized controlled trial. Working group for the study of patient self-management of oral anticoagulation. JAMA 1999;281:145-50.

2 Ansell JE, Hughes R. Evolving models of warfarin management: anticoagulation clinics, patient self-monitoring, and patient self-management. Am Heart J 1996;132:1095-100. 